\title{
The dependency of adhesion and friction on electrostatic attraction
}

Cite as: J. Chem. Phys. 148, 144701 (2018); https://doi.org/10.1063/1.5024038

Submitted: 30 January 2018 . Accepted: 26 March 2018 . Published Online: 09 April 2018

B. N. J. Persson

\section{COLLECTIONS}

This paper was selected as Featured
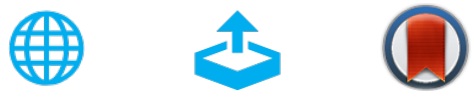

\section{ARTICLES YOU MAY BE INTERESTED IN}

Rubber friction: The contribution from the area of real contact

The Journal of Chemical Physics 148, 224701 (2018); https://doi.org/10.1063/1.5037136

Theoretical model offers more complete account of robotic gripping physics

Scilight 2018, 150002 (2018); https://doi.org/10.1063/1.5033422

Adhesion between rubber and glass in dry and lubricated condition

The Journal of Chemical Physics 148, 234702 (2018); https://doi.org/10.1063/1.5025605

PHYSICS TODAY

WHITEPAPERS
ADVANCED LIGHT CURE ADHESIVES

Take a closer look at what these

environmentally friendly adhesive

systems can do
READ NOW

PRESENTED BY Q MASTERBOND: 


\title{
The dependency of adhesion and friction on electrostatic attraction
}

\author{
B. N. J. Persson a) \\ PGI-1, FZ Jülich, Jülich, Germany
}

(Received 30 January 2018; accepted 26 March 2018; published online 9 April 2018)

\begin{abstract}
I develop a general mean-field theory for the influence of electrostatic attraction between two solids on the contact mechanics. I assume elastic solids with random surface roughness. I consider two cases, namely, with and without an electrically insulating layer between the conducting solids. The former case is important for, e.g., the finger-touch screen interaction. I study how the electrostatic attraction influences the adhesion and friction. For the case of an insulating layer, I find that when the applied nominal contact pressure is relatively small, as the applied voltage increases, there is a sharp increase in the contact area, and hence in the friction, at a critical voltage. Published by AIP Publishing. https://doi.org/10.1063/1.5024038
\end{abstract}

\section{INTRODUCTION}

Sliding friction depends sensitively on the nature of the materials involved, in particular, at the sliding interface where the surface topography, contamination films, and the atomic and molecular nature of the contacting surfaces strongly influence the friction. ${ }^{1-3}$ However, the sliding friction also depends on external conditions such as temperatures and the humidity and on mechanical vibrations and electric fields. Thus it has been shown that ultrasonic vibrations act to reduce friction, e.g., between the finger and a counter surface. ${ }^{4}$ Similarly, an applied electric voltage between two solids often result in the accumulation of charges of opposite sign on the surfaces at the contacting interface. This results in an electrostatic attraction, denoted electroadhesion, which adds to the external load (squeezing-force) and increases the area of contact and the sliding friction force. This effect is now intensively studied, e.g., for grippers for robotics, ${ }^{5}$ and in the context of touch screen applications, where one is interested in the friction between a finger and the touch screen. ${ }^{6-8}$ These types of displays have seen a wide variety of applications such as increasing the physicality of touch interaction or for influencing shape perception.

The term electroadhesion is drawn from the 1923 work of Danish scientists Johnsen and Rahbek. ${ }^{9}$ Working with polished lithographic stone and metal surfaces, this term was used to describe the physical phenomenon of considerable adhesion which developed when the highly resistive stone was placed on top of a metal plate and a high voltage was applied between them.

I develop a general mean-field theory for the influence of electrostatic attraction between two solids on the contact mechanics. I consider first the limiting case when an electric insulating film separate two conducting bodies and where the total voltage drop occurs over the insulating film. This may never hold strictly in reality since all materials have a finite electric conductivity. Still if the contact time is short enough, the leak current results in negligible charge transfer, and in this

a)URL: www.MultiscaleConsulting.com. case assuming a perfectly insulating material is a very good approximation.

Next I assume two electric conducting solids. Because of surface roughness, the contact between the solids is never perfect. This will result in a contact resistance and in voltage drop $\Delta V$ over a narrow region at the interface, which in general is smaller than the applied voltage $V$. Hence, in this case too, there will be an electric field in the non-contact region between the solids which result in electroadhesion. Here I note that for elastic solids the contact resistance is proportional to the mechanical contact stiffness, a fundamental result first derived by Barber ${ }^{10}$ (see also Refs. 11 and 12). We will make use of this result below where the contact stiffness and other contact mechanics properties are obtained using the Persson $e t$ al. contact mechanics theory. ${ }^{12-17}$

In the literature, there is a discussion about the mathematical relation between the adhesion (and friction) force and the applied voltage. Several different equations have been presented and there is no general consensus about which one is most accurate. ${ }^{18}$ In this paper, I consider this problem in greater detail. I assume that the interfacial separation $u(x, y)$ is arbitrary, except I make the small-slope approximation, i.e., I assume $|\nabla u|<1$. The small-slope approximation is usually used in fluid dynamics at interfaces, where it results in a huge simplification of the Navier Stokes equations (which reduces to the Reynolds equation when $|\nabla u| \ll 1$ ).

This paper is organized as follows: In Secs. II and III, using the small-slope approximation, I derive expressions for the average electroadhesive stress for the two cases discussed above. Since the interfacial separation $u(x, y)$ depends on the attractive electrostatic stress, in Sec. IV I present a mean-field treatment of the electroadhesion force, where $u(x, y)$ is modified by the adhesion stress. In Sec. V I present numerical results. Section VI contains a discussion and Sec. VII contains the summary and conclusion.

\section{ELECTROSTATIC ATTRACTION: INSULATING SOLIDS}

We consider the contact configuration shown in Fig. 1. We assume the solids make atomic contact in a fraction $A / A_{0}$ of the 


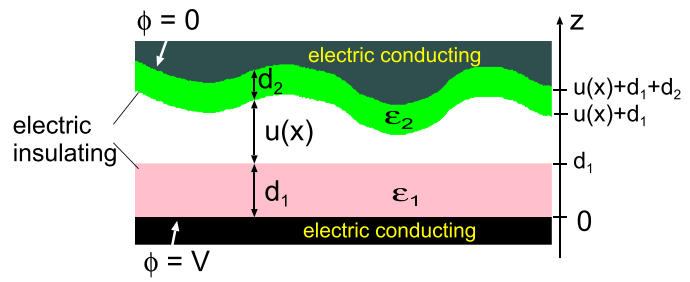

FIG. 1. An elastic solid with surface roughness above a rigid solid with a flat surface. Both solids are conducting materials with insulating surface layers of thickness $d_{1}$ and $d_{2}$ and dielectric constants $\epsilon_{1}$ and $\epsilon_{2}$. An electric voltage difference $V$ occurs between the two conducting solids. The interfacial separation $u=u(\mathbf{x})$ depends on the lateral coordinate $\mathbf{x}=(x, y)$.

nominal contact area $A_{0}$ (in the figure $A=0$ ). The non-contact region is filled with air, but we can consider this region as vacuum because the dielectric constant of air $\left(\epsilon_{\text {air }} \approx 1.00059\right)$ is nearly the same as that of vacuum $(\epsilon=1)$.

The electrostatic potential satisfies $\nabla^{2} \phi=0$ everywhere except at the different interfaces where the material properties change. The solids at the interface have surface roughness given by the functions $h_{1}(\mathbf{x})$ and $h_{2}(\mathbf{x})$ before contact (undeformed height profiles), where $\mathbf{x}=(x, y)$ denote the position vector in the $x y$-plane. We define the combined height profile $h(\mathbf{x})=h_{1}-h_{2}$. Here we will make the small slope approximation $|\nabla h| \ll 1$. When the solids are in contact, the interfacial separation will be denoted by $z=u(\mathbf{x})$ which differ from $h(\mathbf{x})$ because of elastic deformation of the solids. Assuming the small slope approximation case, we can approximate

$$
\nabla^{2} \phi \approx \frac{\partial^{2} \phi}{\partial z^{2}} \approx 0
$$

In this case, we can write the electric potential as (see Fig. 1)

$$
\begin{gathered}
\phi=V+b_{1} z \text { for } 0<z<d_{1}, \\
\phi=a+b\left(z-d_{1}\right) \text { for } d_{1}<z<u(\mathbf{x})+d_{1}, \\
\phi=b_{2}\left(z-u(\mathbf{x})-d_{1}-d_{2}\right) \text { for } u(\mathbf{x})+d_{1}<z<u(\mathbf{x})+d_{1}+d_{2} .
\end{gathered}
$$

Note that $\phi=V$ for $z=0$ and $\phi=0$ for $z=u(\mathbf{x})+d_{1}+d_{2}$. At the two solid-vacuum interfaces $z=d_{1}$ and $z=u(\mathbf{x})+d_{1}$, the electric potential $\phi$ and $\epsilon E_{z}$ (where $E_{z}=-\partial \phi / \partial z$ is the $z$-component of the electric field) must be continuous. This gives the equations

$$
\begin{gathered}
a=V+b_{1} d_{1}, \quad a+b u=-b_{2} d_{2}, \\
b=\epsilon_{1} b_{1}, \quad b=\epsilon_{2} b_{2} .
\end{gathered}
$$

From these equations, we get

$$
b=-\frac{V}{u+d_{1} / \epsilon_{1}+d_{2} / \epsilon_{2}} .
$$

The electric field in the vacuum region

$$
E_{z}=-\frac{\partial \phi}{\partial z}=-b=\frac{V}{u+d_{1} / \epsilon_{1}+d_{2} / \epsilon_{2}} .
$$

We will denote $h_{0}=d_{1} / \epsilon_{1}+d_{2} / \epsilon_{2}$ so that

$$
E_{z}(\mathbf{x})=-\frac{V}{u(\mathbf{x})+h_{0}} \text {. }
$$

The normal stress acting on the surfaces at the solid-vacuum interfaces is given by the $z z$ component of the Maxwell stress tensor which gives

$$
\sigma_{z z}(\mathbf{x})=\frac{\epsilon_{0}}{2} E_{z}^{2}=\frac{\epsilon_{0}}{2}\left(\frac{V}{u(\mathbf{x})+h_{0}}\right)^{2} .
$$

We are interested in the stress averaged over the surface roughness. Let $P(p, u)$ be the probability distribution of interfacial separations (see Ref. 17) so that

$$
\left\langle\sigma_{z z}\right\rangle=\frac{\epsilon_{0}}{2} V^{2} \int_{0}^{\infty} d u P(p, u) \frac{1}{\left(u+h_{0}\right)^{2}} .
$$

We can write

$$
P(p, u)=\frac{A}{A_{0}} \delta(u)+P_{1}(p, u),
$$

where $A / A_{0}$ is the relative contact area and where $P_{1}(p, u)$ is normalized so that

$$
\int_{0}^{\infty} d u P_{1}(p, u)=1-\frac{A}{A_{0}} .
$$

Thus we can write

$$
\left\langle\sigma_{z z}\right\rangle=\frac{\epsilon_{0}}{2} V^{2}\left[\frac{1}{h_{0}^{2}} \frac{A}{A_{0}}+\int_{0}^{\infty} d u P_{1}(p, u) \frac{1}{\left(u+h_{0}\right)^{2}}\right] .
$$

The study above can be easily extended to the case where the applied voltage $V$ depends on time, $V=V(t)$. If we define the Fourier transform

$$
V(\omega)=\frac{1}{2 \pi} \int d t V(t) e^{i \omega t}
$$

so that

$$
V(t)=\int d \omega V(\omega) e^{-i \omega t} .
$$

Then the derivation above is unchanged and gives

$$
E_{z}(\omega, \mathbf{x})=-\frac{V(\omega)}{u(\mathbf{x})+h_{0}(\omega)},
$$

where

$$
h_{0}(\omega)=\frac{d_{1}}{\epsilon_{1}(\omega)}+\frac{d_{2}}{\epsilon_{2}(\omega)},
$$

where $\epsilon_{1}(\omega)$ and $\epsilon_{2}(\omega)$ are the dielectric functions of the two media. For example, if $V(t)=V_{0} \cos \omega_{0}$, then

$$
V(\omega)=\frac{1}{2} V_{0}\left[\delta\left(\omega+\omega_{0}\right)+\delta\left(\omega-\omega_{0}\right)\right]
$$

and

$$
\begin{aligned}
E_{z}(t, \mathbf{x}) & =-\int d \omega \frac{V(\omega)}{u(\mathbf{x})+h_{0}(\omega)} e^{-i \omega t} \\
& =\frac{1}{2} V_{0}\left[\frac{e^{-i \omega_{0} t}}{u(\mathbf{x})+h_{0}\left(\omega_{0}\right)}+\frac{e^{i \omega_{0} t}}{u(\mathbf{x})+h_{0}\left(-\omega_{0}\right)}\right] \\
& =V_{0} \operatorname{Re}\left[\frac{e^{-i \omega_{0} t}}{u(\mathbf{x})+h_{0}\left(\omega_{0}\right)}\right],
\end{aligned}
$$

where we have used that $h_{0}(-\omega)=h_{0}^{*}(\omega)$. If we define the angle $\phi$ via

$$
u(\mathbf{x})+h_{0}\left(\omega_{0}\right)=\left|u(\mathbf{x})+h_{0}\left(\omega_{0}\right)\right| e^{i \phi},
$$

we can write

$$
E_{z}(t, \mathbf{x})=V_{0} \frac{\cos \left(\omega_{0} t+\phi\right)}{\left|u(\mathbf{x})+h_{0}\left(\omega_{0}\right)\right|} .
$$


The $z z$-component of the stress tensor becomes

$$
\begin{aligned}
\sigma_{z z}(t, \mathbf{x}) & =\frac{\epsilon_{0}}{2} \frac{V_{0}^{2}}{2}\left(\frac{1}{\left|u(\mathbf{x})+h_{0}\right|^{2}}+\operatorname{Re}\left[\frac{e^{-2 i \omega_{0} t}}{\left(u(\mathbf{x})+h_{0}\right)^{2}}\right]\right) \\
& =\frac{1}{4} \epsilon_{0} V_{0}^{2} \frac{1+\cos \left(2 \omega_{0} t+2 \phi\right)}{\left|u(\mathbf{x})+h_{0}\left(\omega_{0}\right)\right|^{2}} .
\end{aligned}
$$

The normal stress averaged over the surface roughness becomes

$$
\left\langle\sigma_{z z}\right\rangle=\frac{1}{4} \epsilon_{0} V_{0}^{2} \int_{0}^{\infty} d u P(p, u) \frac{1+\cos \left(2 \omega_{0} t+2 \phi\right)}{\left|u+h_{0}\left(\omega_{0}\right)\right|^{2}} .
$$

\section{ELECTROSTATIC ATTRACTION: CONDUCTING SOLIDS}

In Sec. II, we assumed that the solids at the interface are electric insulators which can be described in the relevant frequency range by a real dielectric function $\epsilon(\omega)$. However most solids have a non-zero electric conductivity $\kappa$. In this case, one needs in general to take into account that when an electric voltage is applied between the solids, an electric current will flow through the asperity contact regions and the voltage drop $\Delta V$ over the contacting interface will depend on the electric conductivities $\kappa_{1}$ and $\kappa_{2}$ of the solids and on the contact resistance (see below). In this section, we will consider this problem in some detail.

Consider a slab of thickness $d$ of a material with the electric conductivity $\kappa$. Assume a voltage $V$ acts between the two ends of the slab. This will result in an electric field $E=V / d$ and an electric current density $J=\kappa E$ in the slab. If the electric field is due to a surface charge density $n e$ on the surface $z=0$, then $E=n e / \epsilon \epsilon_{0}$. We define the characteristic time (or relaxation time) $t=\tau$ so that $J \tau=n e$. We can interpret $\tau$ as the time it takes for the surface charge ne to flow from the upper surface to the bottom surface (see Fig. 2). We get $J \tau=\kappa E \tau$ $=\kappa n e \tau / \epsilon \epsilon_{0}$. Using $J \tau=n e$ gives $\tau=\epsilon \epsilon_{0} / \kappa$. If an experiment is performed during a time period $t \ll \tau$, then we can neglect the leakage of electric charge from the upper surface, and in this case, the analysis in Sec. II is valid. However, for times $t \gg \tau$, the charge initially localized to the upper surface will have moved to the surface at the interface. In this section, we assume that the latter case holds.

Figure 3 shows two elastic blocks, with finite electric conductivity, squeezed together with a nominal contact pressure $p_{0}$. If a voltage $V$ is applied between the upper and lower flat surfaces of the two blocks, an electric current $J$

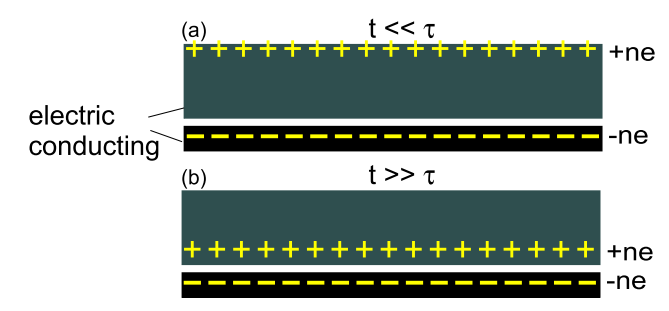

FIG. 2. (a) If the upper slab is a material with the electric conductivity $\kappa$ and the (positive) surface charge ne on the top surface, the charge will flow toward the bottom surface. (b) After a characteristic time of order $\tau=\epsilon \epsilon_{0} / \kappa$, the charge is located on the bottom surface close to the negative charged slab below it.

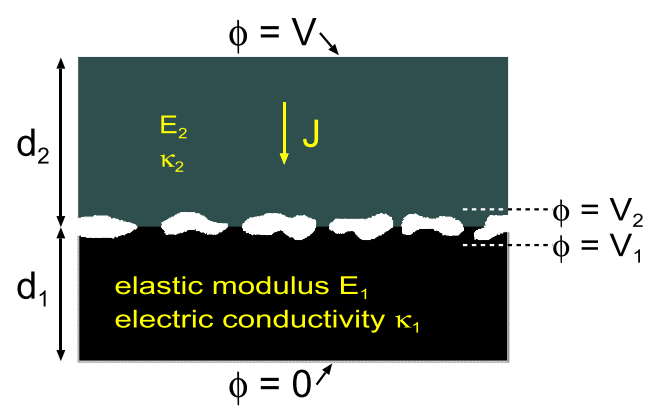

FIG. 3. Two elastic blocks, with finite electric conductivity, squeezed together with a nominal contact pressure $p_{0}$. If a voltage $V$ is applied between the upper and lower flat surfaces of the two blocks, an electric current $J$ will flow from the upper surface to the lower surface. Far away from the contacting interface, the current is uniform in the $x y$-plane but close to the interface highly nonuniform as the current will flow through the area of real contact. A potential drop $\Delta V$ will occur over a narrow region at the interface, which determines the electric contact transfer coefficient $\alpha$ via $J=\alpha \Delta V$.

will flow from the upper surface to the lower surface. Far enough away from the contacting interface, the current is uniform in the $x y$-plane, but close to the interface, it is highly non-uniform as the current will flow through the area of real contact. The distance $\pm \delta$ from the interface where the electric current is approximately uniform in the $x y$-plane is determined by the average separation between the macroasperity contact regions, which depends on the roll-off wavelength of the surface roughness power spectrum. ${ }^{11}$ A potential drop $\Delta V=V_{2}-V_{1}$ will occur over the narrow region of width $\sim 2 \delta$ at the interface, which determines the electric contact conductivity $\alpha$ via $J=\alpha\left(V_{2}-V_{1}\right)$. Since the voltage $\Delta V$ is the quantity which determines the Coulomb attraction between the surfaces, we need to estimate this quantity.

The electric current

$$
J=\kappa_{2}\left(V-V_{2}\right) / d_{2}, \quad J=\kappa_{1}\left(V_{1}-0\right) / d_{1} .
$$

Combining these equations with $J=\alpha\left(V_{2}-V_{1}\right)$ gives

$$
J=\frac{\alpha V}{1+\alpha\left(d_{1} / \kappa_{1}+d_{2} / \kappa_{2}\right)}
$$

and

$$
\Delta V=\frac{V}{1+\alpha\left(d_{1} / \kappa_{1}+d_{2} / \kappa_{2}\right)} .
$$

For the elastic contact between randomly rough surfaces, it has been shown that the electric contact conductivity is $^{10-12}$

$$
\alpha=\frac{2 \kappa}{E^{*}} K_{\perp}
$$

where

$$
K_{\perp}=-\frac{d p_{0}}{d \bar{u}}
$$

is the mechanical contact stiffness and

$$
\frac{1}{E^{*}}=\frac{1-v_{1}^{2}}{E_{1}}+\frac{1-v_{2}^{2}}{E_{2}}
$$

is the effective Young's modulus ( $E_{1}$ and $v_{1}$ are the Young's modulus and Poisson ratio of solid 1 and similar for solid 2), and

$$
1 / \kappa=1 / \kappa_{1}+1 / \kappa_{2}
$$


is the effective electric conductivity ( $\kappa_{1}$ is the electric conductivity of solid 1 and $\kappa_{2}$ is the electric conductivity of solid 2). Substituting (8) into (7) gives

$$
\Delta V=\frac{V}{1+2 d_{0} K_{\perp} / E^{*}},
$$

where

$$
d_{0}=\kappa\left(\frac{d_{1}}{\kappa_{1}}+\frac{d_{2}}{\kappa_{2}}\right)=\frac{\kappa_{1} d_{2}+\kappa_{2} d_{1}}{\kappa_{1}+\kappa_{2}} .
$$

The relation between the applied nominal contact pressure $p_{0}$ and the average interfacial separation $\bar{u}$ can be calculated using the Persson contact mechanics theory. The average interfacial separation was derived in Refs. 14 and 15 for the case when the nominal contact pressure is not too high (i.e., not close to complete contact) and not so small that finite size effects become important (in which case the two solids make contact only at a few of the highest asperities),

$$
p \approx \beta E^{*} \exp \left(-\bar{u} / u_{0}\right)
$$

or

$$
\bar{u} / u_{0}=\log \left(\beta E^{*} / p\right),
$$

where $u_{0} \approx 0.5 h_{r m s}$, where $h_{\mathrm{rms}}$ is the rms roughness of the (combined) surface roughness profile. Thus in this case, we have

$$
K_{\perp}=p_{0} / u_{0}
$$

and

$$
\alpha=\frac{2 \kappa p_{0}}{E^{*} u_{0}}
$$

Using (8) and (9) gives

$$
\Delta V=\frac{V}{1+2 d_{0} p_{0} /\left(E^{*} u_{0}\right)} .
$$

Note that if $d_{1}=d_{2}$ then $d=d_{1}$ and the voltage drop $\Delta V$ over the contacting interface is independent of the electric properties of the material; i.e., it depends only on the surface roughness and the elastic properties of the solids. The same result holds if $\kappa_{1} d_{2} \gg \kappa_{2} d_{1}$ and $d_{2} \gg d_{1}$ or $\kappa_{2} d_{1} \gg \kappa_{1} d_{2}$ and $d_{1} \gg d_{2}$.

\section{MEAN-FIELD ELECTROSTATIC ADHESION THEORY}

In the simplest approach, one includes the electrostatic attraction $p_{\mathrm{a}}=\left\langle\sigma_{z z}\right\rangle$ as a contribution to the external load. Thus we write the effective loading pressure as

$$
p=p_{0}+p_{\mathrm{a}},
$$

where $p_{0}$ is the applied pressure. Intuitively, one expects this approach to be accurate when the interaction force between the surfaces is long-range, and a similar approach has been used for the attraction resulting from capillary bridges ${ }^{19}$ (see also Ref. 20). To calculate $p_{\mathrm{a}}=\left\langle\sigma_{z z}\right\rangle$, we need to know the probability distribution $P(p, u)$. For randomly rough surfaces, the function $P(p, u)$ was calculated approximately in Ref. 17. For the case of insulating solids (Sec. II), we get from (17) and (2)

$$
p=p_{0}+\frac{\epsilon_{0}}{2} V^{2} \int_{0}^{\infty} d u P(p, u) \frac{1}{\left(u+h_{0}\right)^{2}}
$$

which we can also write as

$$
V^{2}=\frac{2\left(p-p_{0}\right) / \epsilon_{0}}{\int_{0}^{\infty} d u P(p, u)\left(u+h_{0}\right)^{-2}}
$$

from which we can easily calculate $V$ as a function of the nominal contact pressure $p$. For conducting solids (Sec. III) including only the surface charge at the interface, we obtain

$$
p=p_{0}+\frac{\epsilon_{0}}{2} \int_{a_{\mathrm{c}}}^{\infty} d u P_{1}(p, u) \frac{\Delta V^{2}}{u^{2}}
$$

or using (12),

$$
p=p_{0}+\frac{\epsilon_{0}}{2} V^{2} \int_{a_{\mathrm{c}}}^{\infty} d u P_{1}(p, u) \frac{1}{u^{2}\left(1+2 d_{0} K_{\perp}(p) / E^{*}\right)^{2}},
$$

where we have indicated that the stiffness $K_{\perp}$ depends on the pressure $p$. Note that in these equations we use the probability distribution $P_{1}(p, u)$ rather than $P(p, u)=P_{1}(p, u)$ $+\left[A(p) / A_{0}\right] \delta(u)$ since we assume that there is no attraction coming from the contact area in the case of conducting materials. From (19), we get

$$
V^{2}=\frac{2\left(p-p_{0}\right)\left(1+2 d_{0} K_{\perp}(p) / E^{*}\right)^{2} / \epsilon_{0}}{\int_{a_{\mathrm{c}}}^{\infty} d u P_{1}(p, u) u^{-2}} .
$$

In (19), $a_{\mathrm{c}}$ is a cut-off distance. In the numerical study below, I use $a_{\mathrm{c}}=10 \mathrm{~nm}$. The region in space where the surface separation is very small (here $u<u_{\mathrm{c}}$ ) forms narrow strips around the area of real contact; we denote these regions as the rim-area. Since the electric potential is continuous in the contact regions, we expect that the potential drop between the surfaces in the rim-area is much smaller than the (average) potential drop $\Delta V$ over the interface. I attribute the cutoff $a_{\mathrm{c}}$ as due to this effect, but at present I am not aware of any theory to predict the optimum $a_{\mathrm{c}}$ to be used in (19).

The relative contact area $A / A_{0}$ can be obtained using

$$
\frac{A}{A_{0}} \approx \frac{\sqrt{ } \pi}{2} \operatorname{erf}\left(\frac{2 p}{h^{\prime} E^{*}}\right),
$$

where $h^{\prime}$ is the surface rms slope of the combined surface roughness profile. This equation is valid for the contact between homogeneous elastic solids with random surface roughness. When $A / A_{0} \ll 1$, Eq. (21) reduces to

$$
\frac{A}{A_{0}} \approx \frac{2 p}{h^{\prime} E^{*}} .
$$

From the knowledge of $A$, we obtain the friction force from

$$
F_{\mathrm{f}}=A \tau_{\mathrm{f}} .
$$

The frictional shear stress $\tau_{\mathrm{f}}$ is usually independent of the asperity contact pressure $p^{*}=p A_{0} / A$ as long as $p^{*}$ is less than a few MPa. As an example, which is of interest for grippers for robotics, for silicone rubber [polydimethylsiloxane (PDMS)] sliding (in complete contact) on a smooth glass surface at the sliding speed $v \approx 1 \mathrm{~mm} / \mathrm{s}$, experiments have shown that $\tau_{\mathrm{f}} \approx 0.1 \mathrm{MPa}$. At the same sliding speed, for other types of rubber, ${ }^{21,22} \tau_{\mathrm{f}} \approx 1-10 \mathrm{MPa}$, and for the human skin, ${ }^{23,24}$ which is of interest for touch screens, $\tau_{\mathrm{f}} \approx 15 \mathrm{MPa}$. This is also similar to what is observed for plastics (polymers below the glass transition temperature) and also as expected from molecular dynamics calculations. ${ }^{25}$ 
In the numerical results presented in Sec. V, we use the full (numerically generated) relation $\bar{u}(p)$ between the average interfacial separation and the nominal contact pressure $p$, as determined by the Persson contact mechanics theory. Similarly, we also use the full expression for the probability distribution $P(p, u)$ of interfacial separations. However, let us first consider the simplest possible approximation for $P(p, u)$, namely, $P(p, u) \approx \delta(u-\bar{u})$, where $\bar{u}(p)$ is the average interfacial separation when the nominal contact pressure equals to $p$. In addition, let us use the $\bar{u}(p)$ relation derived in Ref. 14 for the case when the nominal contact pressure is not too high (i.e., not close to complete contact), and not so small that finite size effects become important (in which case the two solids makes contact only at a few of the highest asperities ${ }^{17}$ ), as given by (14). In this case, (18) reduces to

$$
V^{2}=\left(p-p_{0}\right) \frac{2}{\epsilon_{0}}\left[u_{0} \log \left(\frac{\beta E^{*}}{p}\right)+h_{0}\right]^{2}
$$

and (20) reduces to

$$
V^{2}=\left(p-p_{0}\right) \frac{2}{\epsilon_{0}}\left[\left(u_{0}+2 \frac{d_{0} p}{E^{*}}\right) \log \left(\frac{\beta E^{*}}{p}\right)\right]^{2} .
$$

These equations are in fact not accurate. Thus the average of $1 /\left(u+h_{0}\right)^{2}$ is in most cases very different from $1 /\left(\bar{u}+h_{0}\right)^{2}$; i.e., it is not a good approximation to replace $\left\langle\left(u+h_{0}\right)^{-2}\right\rangle$ with $\left(\langle u\rangle+h_{0}\right)^{-2}$, where $\langle.$.$\rangle stands for averaging using the$ height distribution $P(p, u)$. The difference between the two averaging procedures depends on $h_{0}$, but in finger applications, the former expression is typically $\sim 10$ times (or more) larger than the latter expression. A similar conclusion holds for the approximation (24), where the result depends on the cut-off distance $a_{\mathrm{c}}$.

From the equations above it is clear that for a given surface roughness power spectrum, $V^{2} / E^{*}$ depends only on $p / E^{*}$ and $p_{0} / E^{*}$. Thus, for example, $V^{2} / E^{*}$ as a function of $p / E^{*}$ for $p_{0}=10^{4} \mathrm{~Pa}$ and $E^{*}=10^{6} \mathrm{~Pa}$ is the same curve as $V^{2} / E^{*}$ as a function of $p / E^{*}$ for $p_{0}=10^{5} \mathrm{~Pa}$ and $E^{*}=10^{7} \mathrm{~Pa}$.

\section{NUMERICAL RESULTS}

Let us now present some numerical results. In the calculations, we assume the surface roughness power spectrum shown in Fig. 4. The surface is self-affine-fractal, with the

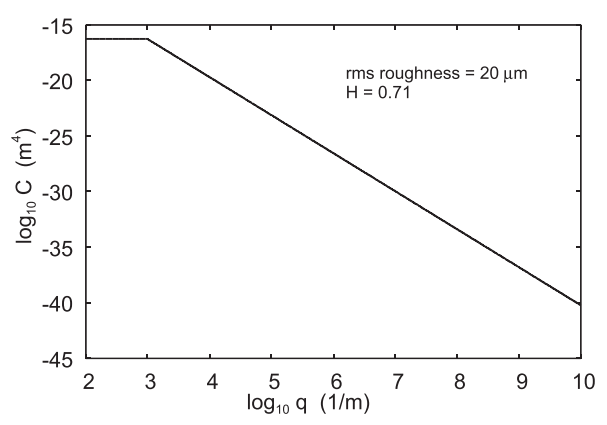

FIG. 4. The surface roughness power spectrum as a function of the wavenumber (log-log scale). The surface is self-affine-fractal, with the rms roughness amplitude $20 \mu \mathrm{m}$ and the Hurst exponent $H=0.71$. rms roughness amplitude $20 \mu \mathrm{m}$ and the Hurst exponent $H=0.71$. We assume the effective thickness of the insulating layer $h_{0}=d_{1} / \epsilon_{1}+d_{2} / \epsilon_{2}=0.3 \mu \mathrm{m}$ and the applied pressure $p_{0}=10 \mathrm{kPa}$.

Figure 5 shows (a) the nominal contact pressure $p$, (b) the average surface separation $\bar{u}$, and (c) the normalized contact area $A / A_{0}$, as a function of the applied voltage $V$ (log-log scale). Results are shown for solids with the effective Young's modulus $E=1 \mathrm{MPa}$ (red curves), $E=10 \mathrm{MPa}$ (blue curves), and $E=100 \mathrm{MPa}$ (pink curves). The red, blue, and green curves are with surface roughness with the power spectrum shown in Fig. 4, while the green line in (a) is for perfectly smooth surfaces (no surface roughness). The open squares in (b) and (c) are the average surface separation and the contact area when $V=0$, respectively, which is determined by the applied pressure $p_{0}=10 \mathrm{kPa}$.

Note that when surface roughness is included, the nominal (or average) contact pressure decreases. This is due to the
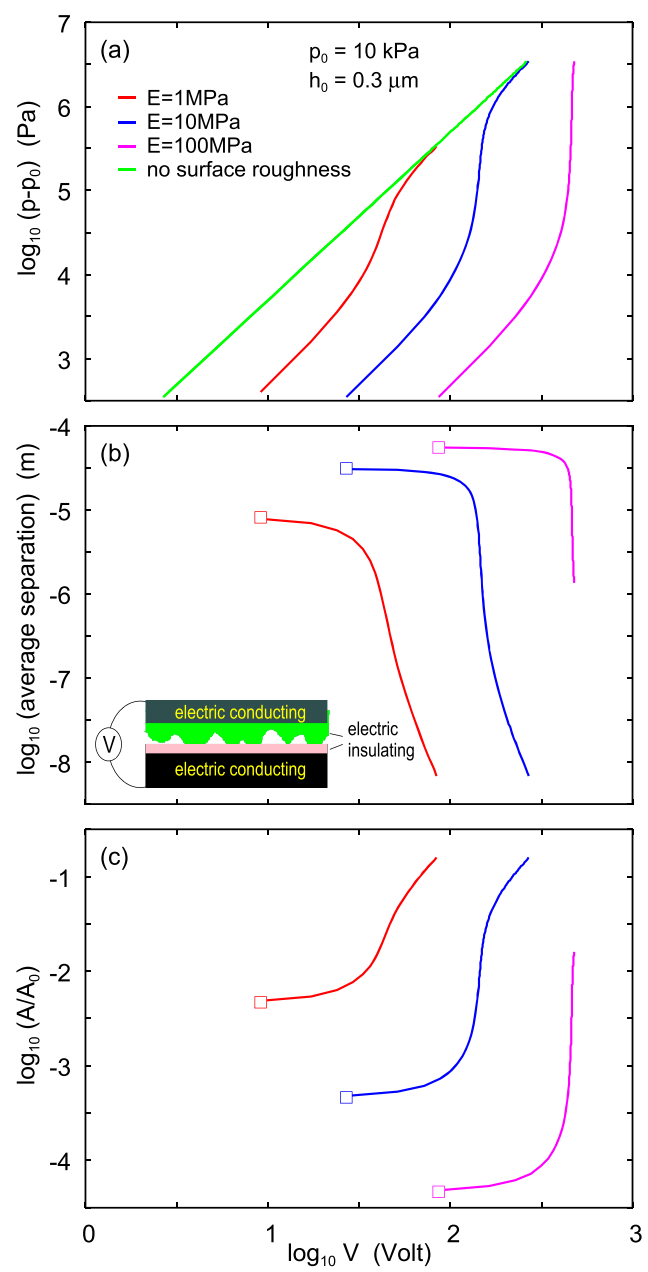

FIG. 5. The nominal contact pressure $p$ (a), the average surface separation $\bar{u}$ (b), and the normalized contact area $A / A_{0}$ (c), as a function of the applied voltage $V$ (log-log scale). The effective thickness of the insulating layer $h_{0}=d_{1} / \epsilon_{1}+d_{2} / \epsilon_{2}=0.3 \mu \mathrm{m}$, and the results are shown for solids with the effective Young's modulus $E=1 \mathrm{MPa}$ (red curves), $E=10 \mathrm{MPa}$ (blue curves), and $E=100 \mathrm{MPa}$ (pink curves). The red, blue, and pink curves are with surface roughness with the power spectrum shown in Fig. 4, while the green line in (a) is for perfectly smooth surfaces (no surface roughness). The open squares in (b) and (c) are the average surface separation and the contact area when $V=0$, respectively, which is determined by the applied pressure $p_{0}=10 \mathrm{kPa}$. 
increase in the separation between the positive and negative charges, which for a given applied voltage $V$ results in a smaller electric field and hence smaller (attractive) electrostatic stress. Note also that for large applied voltage the average surface separation becomes very small, and in this limit, one obtains the same result as for smooth surfaces (green curve). Finally, increasing the elastic modulus increases the surface separation which, for a given applied voltage, reduces the nominal contact pressure, increases the average surface separation, and reduces the contact area.

Figure 6 shows similar results as in Fig. 5 but without the insulating layers. The effective thickness of the conducting layer $d_{0}=\left(\kappa_{1} d_{1}+\kappa_{2} d_{2}\right) /\left(\kappa_{1}+\kappa_{2}\right)=1 \mathrm{~mm}$, and the results are shown for solids with the effective Young's modulus $E=1 \mathrm{MPa}$ (red curves), $E=10 \mathrm{MPa}$ (blue curves), and $E=100 \mathrm{MPa}$ (pink curves). The curves are with surface roughness with the power spectrum shown in Fig. 4, and for the applied nominal contact pressure $p_{0}=10 \mathrm{kPa}$. The dashed
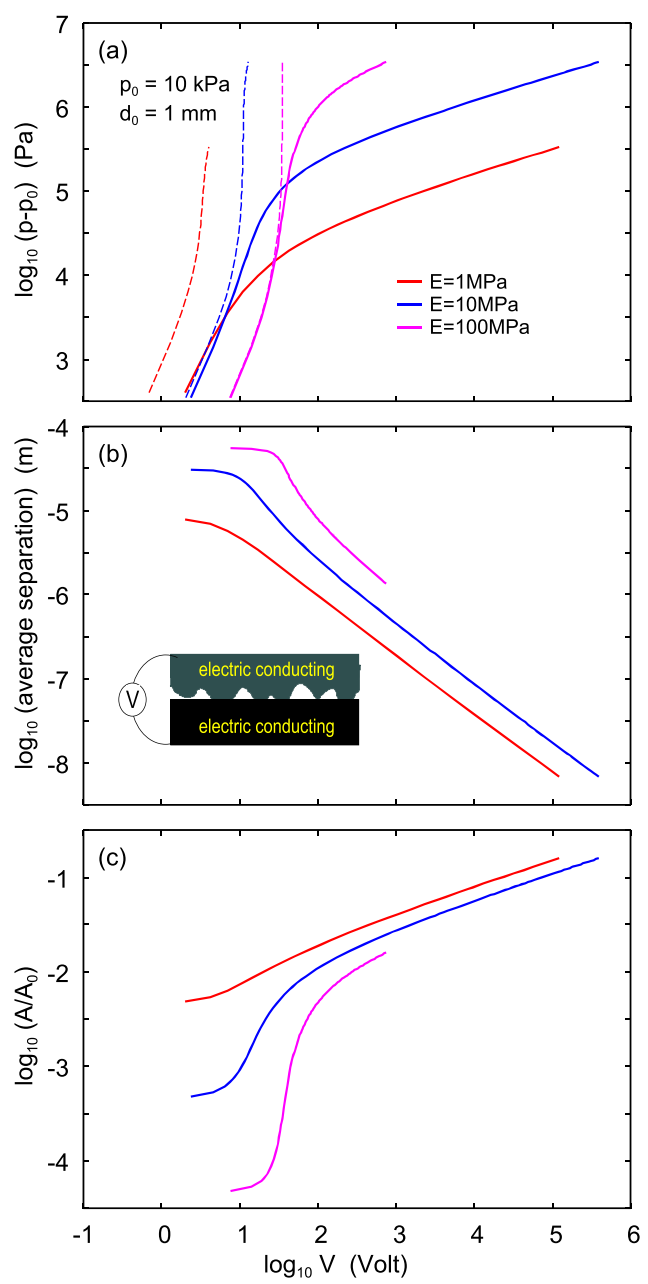

FIG. 6. The nominal contact pressure $p(\mathrm{a})$, the average surface separation $\bar{u}$ (b), and the normalized contact area $A / A_{0}$ (c), as a function of the applied voltage $V$ (log-log scale). The effective thickness of the conducting layer $d_{0}=\left(\kappa_{1} d_{1}+\kappa_{2} d_{2}\right) /\left(\kappa_{1}+\kappa_{2}\right)=1 \mathrm{~mm}$, and the results are shown for solids with the effective Young's modulus $E=1 \mathrm{MPa}$ (red curves), $E=10 \mathrm{MPa}$ (blue curves), and $E=100 \mathrm{MPa}$ (pink curves). The curves are with surface roughness with the power spectrum shown in Fig. 4, and for the applied nominal contact pressure $p_{0}=10 \mathrm{kPa}$. The dashed lines in (a) is assuming the full potential drop $V$ is over the interface. lines in (a) is assuming the full potential drop $V$ is over the interface.

\section{DISCUSSION}

Electroadhesion has recently gained interest because of applications such as touch screens or robotics. Many of these applications involves more complex situations than studied above, e.g., layered and viscoelastic materials such as the human skin, ${ }^{23,24}$ and time varying (usually oscillatory) applied electric potentials, but the theory presented above can easily be extended to these situations. Thus, contact mechanics for layered materials was developed in Ref. 26 and is as simple to implement as homogeneous materials.

Some applications such as grippers for robotics require elastically very soft attachment pads in order to adhere to and move bodies with irregular and complex surface topography. ${ }^{27}$ Only by having soft attachment pads is it possible to generate enough contact area (or surfaces at short separation) to obtain big enough adhesion and friction forces. This is also the case for the attachment pads of living bodies, e.g., tree frogs and geckos.

The theory presented above assumes that the attraction between the solids is due only to the applied voltage. In reality, there will always be other attractive interactions between two contacting solids; e.g., the van der Waals interaction will operate between all solids, and capillary bridges can be very important in some cases, e.g., for the human skin. These additional interactions can also be included in the theory presented above. Thus the role of capillary bridges on adhesion was studied in Ref. 19, and a more general theory of adhesion was developed in Refs. 20 and 28.

In the theory presented above, we have also neglected electrical breakdown across the narrow gap between the contacting solids. ${ }^{29}$ When a large electric potential is applied between narrowly separated surfaces, a very large electric field can prevail, in particular, close to high and sharp asperities. If the local electric field becomes larger than some critical value, breakdown occurs. For gap separations typical in many applications $(\approx 1 \mu \mathrm{m}$ or less), the breakdown voltage is typically a few hundred volts (see Fig. 7). This will affect the results presented in Figs. 5 and 6 for large voltage.

In the study in Sec. II, we have assumed that the insulating film has a constant effective thickness $h_{0}$. In some applications, such as for grippers for robotics or for the finger in the context of touch screen applications, the insulating layer is made from an elastically very soft material. In this case, the electrostatic adhesion will compress the insulating film in the asperity contact regions, and the effective film thickness $h_{0}$ will therefore vary with the spatial coordinate $(x, y)$ on the same length scale as the (lateral variation) of the surface roughness. This effect was not included in the treatment presented in Sec. II, but qualitatively its effect is clear: In the asperity contact regions, where the electrostatic adhesion is strongest, the insulating film is squeezed between two surfaces and will get (locally) thinner, which will increase the electrostatic attraction even more. Associated with this will also be an increased local electric field which could increase the probability for electric breakdown. 


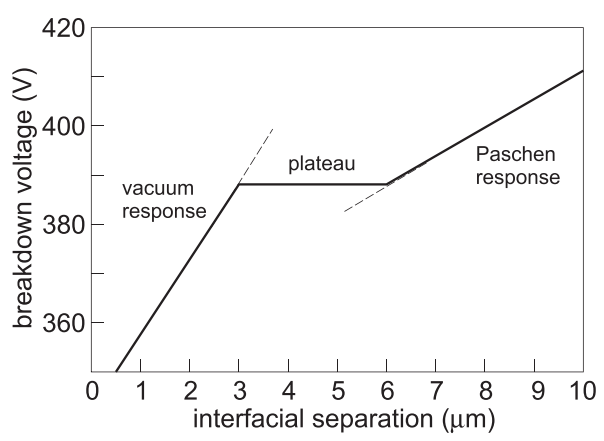

FIG. 7. The breakdown voltage between two silicone surfaces (single gap) as a function of the surface separation. The figure is based on experimental data (see Ref. 29) obtained in the normal atmosphere. The results indicate an avalanche breakdown process in large gaps (Paschen region), with a transition region to small gaps in which vaporization due to the field emission current appears to be the dominant breakdown process (see Ref. 29).

\section{SUMMARY AND CONCLUSION}

I have developed a general mean-field theory for the influence of electrostatic attraction on the contact mechanics between two elastic solids with random surface roughness. I have considered two cases, namely, with and without an electrically insulating layer between the conducting solids. The former case is important for, e.g., the finger-touch screen interaction. I have studied how the electrostatic attraction influences the adhesion and friction. For the case of an insulating layer, I found that when the applied nominal contact pressure is relatively small, as the applied voltage increases, there is a sharp increase in the contact area and hence in the friction at a critical voltage. The effect results from a positive feedback mechanism: increasing the electric voltage result in a larger electric field in the gap between the solids. This pulls the solids in closer contact (elastic deformations) which increases the electric field further, and so on.

\section{ACKNOWLEDGMENTS}

I thank C. Basdogan for drawing my attention to the subject of electroadhesion. I thank him and J. E. Colgate and C. Shultz for many useful discussions and references. This work was performed within a Reinhart-Koselleck project funded by the Deutsche Forschungsgemeinschaft (DFG). I would like to thank DFG for the project support under the reference German Research Foundation DFG-Grant No. MU 1225/36-1.

${ }^{1}$ B. N. J. Persson, Sliding Friction: Physical Principles and Applications (Springer, Heidelberg, 2000).

${ }^{2}$ E. Gnecco and E. Meyer, Elements of Friction Theory and Nanotribology (Cambridge University Press, 2015).

${ }^{3}$ J. N. Israelachvili, Intermolecular and Surface Forces, 3rd ed. (Academic, London, 2011).

${ }^{4}$ M. Wiertlewskia, R. F. Friesena, and J. E. Colgatea, "Partial squeeze film levitation modulates fingertip friction,” Proc. Natl. Acad. Sci. U. S. A. 113, 9210 (2016).
${ }^{5}$ T. Nakamura and A. Yamamoto, "Modeling and control of electroadhesion force in DC voltage," ROBOMECH J. 4, 18 (2017).

${ }^{6}$ Y. Vardar, B. Güclü, and C. Basdogan, "Effect of waveform on tactile perception by electrovibration displayed on touch screens," IEEE Trans. Haptics 10, 488 (2017).

${ }^{7}$ O. Sirin, A. Barrea, P. Lefevre, J.-L. Thonnard, and C. Basdogan, "Experimental evaluation of contact mechanics for electrovibration," (to be published)

${ }^{8}$ D. J. Meyer, M. A. Peshkin, and J. E. Colgate, Fingertip Friction Modulation Due to Electrostatic Attraction [World Haptics Conference (WHC), 2013], pp. 43-48.

${ }^{9}$ A. Johnsen and K. Rahbek, "A physical phenomenon and its applications to telegraphy, telephony, etc.," J. Inst. Electr. Eng. 61, 713-725 (1923).

${ }^{10} \mathrm{~J}$. R. Barber, "Bounds on the electrical resistance between contacting elastic rough bodies," Proc. R. Soc. A 459, 53 (2003).

${ }^{11}$ B. N. J. Persson, B. Lorenz, and A. I. Volokitin, "Heat transfer between elastic solids with randomly rough surfaces," Eur. Phys. J. E 31, 3 (2010).

${ }^{12}$ C. Campana, B. N. J. Persson, and M. H. Müser, "Transverse and normal interfacial stiffness of solids with randomly rough surfaces," J. Phys.: Condens. Matter 23, 085001 (2011).

${ }^{13}$ B. N. J. Persson, "Theory of rubber friction and contact mechanics," J. Chem. Phys. 115, 3840 (2001).

${ }^{14}$ C. Yang and B. N. J. Persson, "Contact mechanics: Contact area and interfacial separation from small contact to full contact," J. Phys.: Condens. Matter 20, 215214 (2008).

${ }^{15}$ B. N. J. Persson, "Relation between interfacial separation and load: A general theory of contact mechanics," Phys. Rev. Lett. 99, 125502 (2007).

${ }^{16}$ L. Pastewka, N. Prodanov, B. Lorenz, M. H. Müser, M. O. Robbins, and B. N. J. Persson, "Finite-size scaling in the interfacial stiffness of rough elastic contacts," Phys. Rev. E 87, 062809 (2013).

${ }^{17}$ A. Almqvist, C. Campana, N. Prodanov, and B. N. J. Persson, "Interfacial separation between elastic solids with randomly rough surfaces: Comparison between theory and numerical techniques," J. Mech. Phys. Solids 59, 2355 (2011).

${ }^{18}$ T. Vodlak, Z. Vidrih, E. Vezzoli, B. Lemaire-Semail, and D. Peric, "Multiphysics modelling and experimental validation of electrovibration based haptic devices," Biotribology 8, 12 (2016).

${ }^{19}$ B. N. J. Persson, "Capillary adhesion between elastic solids with randomly rough surfaces," J. Phys.: Condens. Matter 20, 315007 (2008).

${ }^{20}$ B. N. J. Persson and M. Scaraggi, "Theory of adhesion: Role of surface roughness," J. Chem. Phys. 141, 124701 (2014).

${ }^{21}$ K. G. Rowe, A. I. Bennett, B. A. Krick, and W. G. Sawyer, "In situ thermal measurements of sliding contacts," Tribol. Int. 62, 208 (2013).

${ }^{22}$ B. Lorenz, Y. R. Oh, S. K. Nam, S. H. Jeon, and B. N. J. Persson, "Rubber friction on road surfaces: Experiment and theory for low sliding speeds," J. Chem. Phys. 142, 194701 (2015).

${ }^{23}$ B. N. J. Persson, A. Kovalev, and S. N. Gorb, "Contact mechanics and friction on dry and wet human skin," Tribol. Lett. 50, 17 (2013).

${ }^{24}$ A. E. Kovalev, K. Dening, B. N. J. Persson, and S. N. Gorb, "Surface topography and contact mechanics of dry and wet human skin," Beilstein J. Nanotechnol. 5, 1341 (2014).

${ }^{25}$ I. M. Sivebæk, V. N. Samoilov, and B. N. J. Persson, "Frictional properties of confined polymers," Eur. Phys. J. E 27(1), 37 (2008).

${ }^{26}$ B. N. J. Persson, "Contact mechanics for layered materials with randomly rough surfaces," J. Phys.: Condens. Matter 24, 095008 (2012).

${ }^{27} \mathrm{See}$ https://lmts.epfl.ch/electroadhesion for a movie about electro-adhesion for soft gripper.

${ }^{28}$ B. N. J. Persson, "Adhesion between an elastic body and a randomly rough hard surface,” Eur. Phys. J. E: Soft Matter 8, 385 (2002).

${ }^{29}$ F. W. Strong, J. L. Skinner, P. M. Dentinger, and N. C. Tien, "Electrical breakdown across micron scale gaps in MEMS structures," Proc. SPIE 6111, 611103 (2006). 